\title{
Bivariate Functional Quantile Envelopes with Application to Radiosonde Wind Data
}

\author{
Gaurav Agarwal ${ }^{1}$ and Ying Sun ${ }^{1}$ \\ ${ }^{1}$ CEMSE Division, King Abdullah University of Science and Technology, \\ Thuwal 23955-6900, Saudi Arabia. \\ E-mail: ying.sun@kaust.edu.sa
}

\begin{abstract}
The global radiosonde archives contain valuable weather data, such as temperature, humidity, wind speed, wind direction, and atmospheric pressure. Being the only direct measurement of these variables in the upper air, they are prone to errors. Therefore, a robust analysis and outlier detection of radiosonde data is essential. Among all the variables, the radiosonde winds, which consist of wind speed and direction, are particularly challenging to analyze. In this paper, we treat the wind profiles as bivariate functional data across several pressure levels. Since the bivariate distribution of the components of radiosonde winds at a given pressure level is not Gaussian but instead skewed and heavy-tailed, we propose a set of robust quantile methods to characterize the distribution as well as an outlier detection procedure to identify both magnitude and shape outliers. The proposed methods provide an informative visualization tool for bivariate functional data. We also introduce two methods of predicting this bivariate distribution at unobserved pressure levels. In our simulation study, we show that our methods are robust against different types of outliers and skewed data. Finally, we apply our methods to radiosonde wind data in order to illustrate our proposed quantile analysis methods for visualization, outlier detection, and prediction.
\end{abstract}

Some key words: Bivariate functional data, cubic spline smoothing, kriging, outliers, quantile contours

Short title: Bivaraite functional quantile envelopes

\section{Introduction}

Weather data obtained from the atmosphere, beginning three meters above the earth's surface, is known as weather balloon data or upper air data. A small, expendable instrument known as the radiosonde, which is suspended below a 2-meter wide balloon filled with hydrogen or helium that ascends through the upper-air, collects and transmits that data back to the ground. The sensors on the radiosonde measure vertical profiles of temperature, humidity, atmospheric pressure, and geopotential height. By tracking the position of the radiosonde in flight, information on wind speed and direction is also obtained. 
The Integrated Global Radiosonde Archive (IGRA) consists of more than 1500 globally distributed radiosonde observations from different time periods, ranging from the 1960s to the present; an overview of the dataset is given in Durre et al. (2006). The National Center for Atmospheric Research (NCAR) Upper Air Database (UADB) contains the longest possible time series of radiosonde data, from the 1920 s to the present, at more than 1600 global locations. Analyzing such substantial and complex datasets need a robust analysis. The dataset analyzed in this paper is available online for free at (https://rda.ucar.edu/datasets/ds370.1/) in the Research Data Archive at NCAR (DSS/CISL/NCAR/UCAR, 2014).

Radiosonde data has been extensively used in weather and climate research activities (Chien and Smith, 1973) and studying the features of global warming (Sherwood and Nishant, 2015). The main use of radiosonde data is to input them into climate models for numerical weather predictions. The radiosonde winds were used as data assimilation products in the National Center for Environmental Prediction reanalyses (Kalnay et al., 1996; Kanamitsu et al., 2002). Sherwood et al. (2008) emphasized the utility of wind data in explaining climatological variations, and Hollingsworth and Lönnberg (1986) used radiosonde data to predict wind forecast errors, which were used as an input to a global data assimilation system. So the quality control of weather data is of vital importance. The lack of quality control of the data causes hindrance to the quality of the analysis.

The wind speed and direction from the radiosonde data are recorded in terms of orthogonal velocity components, i.e., u-component and v-component, along different pressure levels, where $u$ represents the component of wind speed towards the east, and $v$ indicates the component of wind speed towards the north. In fact, the $u$ and $v$ components are the cartesian coordinates of the wind speed and direction transformed from their polar coordinates. Typically, for radiosonde data, the bivariate distribution of these two components of wind is non-Gaussian, heavy-tailed, skewed, and susceptible to both measurement and random errors. Hence, robust methods are needed to analyze such distributions. Therefore, in our paper, we study the bivariate distribution of 
these two components along different pressure levels. We treat the $u$ and $v$ components of wind as bivariate functions of the pressure level and develop robust methods based on quantiles for the radiosonde wind data analysis.

In practice, quantiles can be used to characterize univariate non-Gaussian distributions nonparametrically. While measures based on the mean are sensitive to outliers, the quantile approach is more robust (Moors et al., 1996). The univariate quantile is well-defined because the ordering of observations is straightforward and follows the natural ordering in the real line ${ }^{\mathbb{R}}$; but there is no such natural basis in the case of multivariate data. It was not until the late 1970s, Barnett (1976) made attempts to define principles for ordering multivariate data that were analogous to the concepts behind univariate order statistics. Chaudhuri (1996) extended the concept of quantiles to multiple dimensions using the geometry, based on Euclidean distances, of the multivariate data. Chakraborty (2003) defined regression quantiles for multivariate quantile regression. Moreover, data depth, introduced by Tukey (1975), provides a way to order multivariate data. Tukey (1975) ordered multivariate data by defining a useful tool of the depth function, known as the Tukey or halfspace depth, which provides a center-outward ranking of data points. Many notions of data depth have been defined since its first introduction, such as the Oja depth (Oja, 1983), simplicial depth (Liu, 1990), projection depth (Zuo and Serfling, 2000; Zuo, 2003), and spatial depth (Vardi and Zhang, 2000; Serfling, 2002).

Kong and Mizera (2012) defined directional quantile envelopes as the intersection of the halfspaces generated from directional quantiles, i.e., quantiles of projections along multiple directions obtained simultaneously, and showed that they are essentially halfspace (Tukey) depth contours. Tukey depth contours completely characterize the empirical distribution of multivariate datasets, as shown in (Struyf and Rousseeuw, 1999) and (Kong and Zuo, 2010). Thus, the directional quantile envelopes provide valuable insights into the multivariate data and are of vital importance. Hallin et al. (2010) defined multivariate quantiles based on the directional approach of Koenker and Bassett's traditional regression quantiles (Koenker and 
Bassett Jr, 1982) and used quantile contour plots to study the properties of underlying multivariate distributions, see also Chakraborty (2001). The previous methods are for multivariate data, and we extend the existing approaches to multivariate functional data.

Data is often available in the form of random curves that may be observed as univariate or multivariate functional data in various fields. For instance, in medical science, it can be the growth curves of patients; in meteorology, weather parameters can be observed along several pressure levels, and in chemometrics, the curves arising from physics and chemistry experiments. Depth notions have been extended to both univariate functional data (Fraiman and Muniz, 2001; López-Pintado and Romo, 2009, 2011) and multivariate functional data Claeskens et al., 2014; López-Pintado et al., 2014; Hubert et al., 2015. Although those depth notions for multivariate functional data are convenient to rank multivariate functions from the center outwards so that the central region can be defined, it is not easy to construct meaningful contours marginally. Since for the radiosonde application, the marginal characterization of the bivariate distribution is also of interest, especially when the observations are not viewed as an entire launch across all the pressure levels, we chose to use the quantile envelope for marginal distributions and connect them across pressure levels afterward.

However, with multivariate functional data, the data complexity increases, and it is even more challenging to visualize the data. For univariate functional data, the functional boxplot (Sun and Genton, 2011) and the adjusted functional boxplot (Sun and Genton, 2012) were defined, which uses the depth to order data and the empirical rule to detect outliers. For multivariate functional data Hubert et al. (2015) used depth functions and distance measures to detect multivariate functional outliers and Dai and Genton (2019, 2018a, b) used multivariate directional outlyingness for visualization and marking outliers. Kong et al. (2015) defined a bivariate quantile model for functional responses for given covariates of interest and constructed directional quantile envelopes to study the quantile association between responses and covariates. Wei (2008) proposed dynamic bivariate 
quantile contours that are flexible to incorporate time-dependent and covariate-varying effect. Kong and Mizera (2012) used the extremal quantile envelopes to detect outliers, though not for functional data. The current statistical analysis for radiosonde data has been limited to multivariate analysis. For radiosonde data, Sun et al. (2017) used contour approaches based on the bivariate skew-t distribution to mark potential outliers at each pressure level. Since the observations can be treated as functional data along the pressure level, we develop a new set of quantile based methods to visualize the data and detect potential outliers.

Here, our primary goal is to estimate and predict bivariate non-Gaussian distributions of bivariate functional data nonparametrically, as well as to detect outliers. We improve on the existing approaches for multivariate data to define methods for multivariate functional data. Due to the increasing need for statistical techniques in functional data analysis, we intend to develop directional quantile envelope methods and an effective visualization tool to provide a graphical representation of the bivariate functional data, which will demonstrate features of the data that might not be apparent otherwise. The existing methods for multivariate functional data, such as multivariate functional depth notions can not be directly applied to construct meaningful contours marginally. Motivated by the heavily-tailed and skewed bivariate radiosonde wind profiles obtained at several atmospheric pressure levels, we propose a model for estimating the directional quantile envelopes of bivariate functional data, visualizing the geometry of the distribution, and detecting both magnitude and shape outliers. We also propose robust methods of predicting the directional quantile envelopes at an unobserved pressure level for the radiosonde winds, using the methods of quantile regression and quantile kriging. The proposed methods provide a robust prediction for the center of the distribution over the traditional predictor, the conditional mean.

The rest of our paper is organized as follows. Section 2 describes the details of directional quantile envelopes and the proposed model for bivariate functional data. We discuss the visualization techniques and methods of detecting outliers, and also describe the two methods used to predict the 
directional quantile envelopes. In Section 3, we use two simulation designs to show the robustness of the proposed methods against outliers and skewed data. In Section 4, we apply these methods to the radiosonde wind data. Finally, Section 5 contains discussions and conclusions.

\section{Methodology}

\subsection{Directional Quantile Envelopes for Bivariate Functional Data}

In this section, we first review the directional quantile envelopes defined by Kong and Mizera (2012) and discuss their useful properties. Then, we define the model used to estimate and predict the directional quantile envelopes for bivariate functional data. We also discuss the estimation of extreme quantile envelopes for outlier detection.

\subsubsection{Directional quantile envelopes}

To characterize the distribution of multivariate data with quantiles, Kong and Mizera (2012) defined directional quantile envelopes. For a random vector $X$, with distribution $F$, they considered a directional approach to project this vector on a straight line and then obtain the quantiles, hence called them directional quantiles. For a direction $s \in \mathbb{R} \mathbb{R}$, belonging to the unit sphere $\mathcal{S}^{d-1}=\left\{y \in \mathbb{R}^{d}:\|\boldsymbol{y}\|=1\right\}$, and quantile level $\tau \in(0,1)$, the $\tau^{\text {th }}$ directional quantile is defined as

$$
Q_{(\tau, s)}(X)=\inf \left\{u: \mathbb{P}_{\left.\left[s^{T} X \leq u\right] \geq \tau\right\}} .\right.
$$

The directional quantile relates to the hyperplane, with the equation of hyperplane ${ }^{T} x=Q_{(x, s)}(x)$, which leads to the characterization of closed halfspace, which is denoted by ${ }^{H\left(s, Q_{(\tau, s)}(x)\right)}=\left\{x: s^{T} x \geq Q_{(\tau, s)}(x)\right\}$. For a fixed $\tau \in(0,1 / 2]$, the directional quantile envelope is generated by taking the intersection of all the halfspaces taken in all possible directions of a subset of $\mathcal{S}^{d-1}$. Then, the $\tau^{\text {th }}$ directional quantile envelope is defined as $D(\tau)=\bigcap_{s \in \mathcal{S}^{\mathcal{S}-1}} H\left(s, Q_{(\tau, s)}(\boldsymbol{x})\right)$. 
The directional quantile envelopes are related to the halfspace depth contours, associated with Tukey. The Tukey depth of a point $x \in \mathbb{R}^{d}$, with respect to the distribution $P$ of the vector $X$, is given as the smallest probability obtained such that any closed halfspace contains $x$, i.e.,

$\mathrm{D}_{\text {Tukey }}(\boldsymbol{x} \mid P)=\inf _{\boldsymbol{u} \in \mathcal{S}^{d-1}} P\left(\boldsymbol{u}^{T} \boldsymbol{x} \geq \boldsymbol{u}^{T} \boldsymbol{X}\right)$

The directional quantile envelopes are essentially Tukey depth level sets, which means contours of equal depth. Since the depth contours characterize the entire underlying multivariate distribution, the directional quantile envelopes describe the the empirical distribution of the multivariate data. In other words, these quantile envelopes give a geometrical representation of multivariate data with a distribution $F$.

In our proposal, we deal with functional data, where the radiosonde wind is treated as a continuous function in pressure level, and for a given pressure level, the two components of the wind have a bivariate distribution marginally. Although we use the method in Kong and Mizera (2012) to construct directional quantile envelopes, the functional aspect allows us to connect or predict those quantiles continuously along pressure levels.

\subsubsection{Directional quantile envelope models for bivariate functional data}

We extend the concept of directional quantile envelopes to the bivariate functional data setup. In practical situations, functional data is usually generated in a discretized form, and is denoted by $\left(X\left(t_{1}\right), \ldots, X\left(t_{m}\right)\right)$. We introduce a model that we will use to estimate the directional quantile envelopes for bivariate functional data. We consider the bivariate functional data, $\boldsymbol{X}(t)=\left(X_{1}(t), X_{2}(t)\right)^{T}$, where ${ }^{t=t_{1}, \ldots, t_{m}}$, belong to an interval ${ }^{\mathcal{T}}$ in ${ }^{\mathbb{R}}$. For a given $t$ and fixed $\tau \in(0,1 / 2]$, we model the directional quantile in the direction $s$ as

$Q_{(\tau, s)}(X(t))=\beta_{0(\tau, s)}+b_{(\tau, s)}(t)$, 
where $^{b_{(\tau, s)}(t)}$ is the cubic spline function given by $b_{(\tau, s)}(t)=\beta_{1(\tau, s)} t+\beta_{2(\tau, s)} t^{2}+\beta_{3(\tau, s)} t^{3}+\sum_{j=1}^{K} \delta_{j(\tau, s)}\left(t-k_{j}\right)_{+}^{3}$, and $\left\{k_{j}\right\}_{j=1}^{K}$ are prespecified set of knots. The knots are typically chosen as suitable quantiles of $t$, as in spline smoothing. Then, we model the directional quantile for all possible directions of the subset of ${ }^{\mathcal{S}^{d-1}}$. Therefore, for each direction $\boldsymbol{s}$, the estimated directional quantile relates to the halfspace ${ }^{H\left(s, Q_{(\tau, s)}(x(t))\right)}=$ $\left\{x: s^{T} x \geq Q_{(r, s)}(x(t))\right\}$, and the estimated directional quantile envelopes for the bivariate functional data at $t$ are given by

$$
D_{t}(\tau)=\bigcap_{s \in \mathcal{S}^{d-1}} H\left(s, Q_{(\tau, s)}(x(t))\right) .
$$

We call ${ }^{D_{t}(\tau)}$ the marginal directional quantile envelopes, and define the marginal median envelope as the one corresponding to the highest $\tau$ because the point corresponding to highest depth value is the deepest (Tukey, 1975). Then, the marginal median is defined as the average of the vertices of the median envelope, and the functional median is defined by connecting the marginal medians at all $t$. Although the directional quantile envelopes can be estimated for any dimension $d \geq 2$, for our application and visualization purposes, we only consider the two-dimensional case, which can be easily visualized.

For the bivariate functional data observed at $t_{1}, \ldots, t_{m}$, the parameters in the model based on the spline (1) are estimated by quantile regression at a given $\tau$ for all $\boldsymbol{s}$. Then, the $\tau^{\text {th }}$ directional quantile envelopes, which we call predicted functional directional quantile envelopes, can be constructed for any $t$. For the bivariate case, we uniformly choose 100 directions along the unit circle. The parameters in the quantile regression model (1) are estimated using all the data for each direction, but the predicted directional quantile envelopes are constructed for a given $t$ using the estimated parameters. Then, the predicted directional quantile generates a halfspace for each direction. We take the intersection of these halfspaces, along 100 directions, and the polygon formed 
from the vertices of the intersecting lines forms the predicted directional quantile envelope.

\subsubsection{Extremal quantile envelopes}

In general, because of insufficient data in the tails of a distribution, the model for directional quantile envelopes for very small values of $T$, e.g., 0.001 for 100 observations, will not be a good fit. Similar to Kong and Mizera (2012), for very small values of $T$, we fit a generalized Pareto distribution (GPD) to the tails of the distribution of $s^{T} X$, for each direction $s$ and each $t$, and obtain the estimate of the prescribed directional quantiles. For the projected distribution $s^{T} X$, the GPD with shape parameter $\xi$ and scale parameter $\beta$ is given by $\tau=F_{P s}\left(s^{T} x\right)=\left\{\begin{array}{l}1-\left(1-\frac{\xi s^{T} x}{\beta}\right)^{1 / \xi}, \quad \xi \neq 0, \\ 1-\exp \left(\frac{-s^{T} x}{\beta}\right), \quad \xi=0 .\end{array}\right.$

We consider 1000 directions along a unit circle. For each direction $\boldsymbol{s}$, we choose the $95^{\text {th }}$ quantile of $s^{T} x$ as the threshold and fit GPD to all exceedances. We estimate the parameters of the distribution using the method of maximum likelihood. After fitting the distribution, the directional extremal quantiles at $t$ are estimated by

$q_{(s, t)}(\tau)=\left\{\begin{array}{l}\hat{\beta}_{(s, t)}\left\{1-(1-\tau) \hat{\xi}_{t}\right\} / \hat{\xi}_{(s, t)}, \quad \hat{\xi}_{(s, t)} \neq 0, \\ -\hat{\beta}_{(s, t)} \log (1-\tau), \quad \hat{\xi}_{(s, t)}=0,\end{array}\right.$

where $\hat{\xi}_{(s, t)}$ and $\hat{\beta}_{(s, t)}$ are the estimated shape and scale parameters, respectively. For each $t$, the extremal quantile envelopes are estimated as the intersection of the halfspaces generated by the estimated directional extremal quantiles from the fitted GPD.

\subsection{Outlier Detection and Visualization}

We detect outliers using the extreme quantile envelopes described above and demonstrate it using the radiosonde wind data. We visualize the vertical 
profiles of the radiosonde wind across several atmospheric pressure levels using the estimated directional quantile envelopes, extreme quantile envelopes, and the estimated median. We devise a method of connecting the envelopes along pressure levels to characterize the changes. We also define two methods for functional outlier detection.

\subsubsection{Detection of outliers}

The radiosonde wind data has two components of wind, $u$-component and $v$ component, measured in meters per second $(\mathrm{m} / \mathrm{s})$, the components of wind towards east and north, respectively. These components of the wind are measured along eight standard atmospheric pressure levels: 70, 100, 200, $250,300,400,500$, and 700 hectopascals (hPa). The extreme quantile envelopes do not have the functional feature; however, we introduce it to detect bivariate outliers that are often of interest in radiosonde data, rather than the entire launch. The wind components were measured at each level directly from the atmosphere and, hence, the measurements are prone to outliers for many reasons, such as human error and data preprocessing errors. We estimate the extreme quantile envelopes for $\tau=0.0002$ at each pressure level. The observations lying outside these extreme quantile envelopes are marked as bivariate outliers. The estimated extreme quantile envelopes for all eight pressure levels of radiosonde wind data at quantile level $\tau=0.0002$ are shown in Figure 1. The bivariate outliers at each pressure level are marked by red dots. The outliers were in the range of $0.06 \%$ to $0.11 \%$ for all pressure levels.

\subsubsection{Visualization of quantile envelopes}

We consider the two components of wind across pressure levels to be discretized bivariate functional data. Using the model and the estimation procedure defined in Section 2.1.2, we estimate the directional quantile envelopes of these bivariate functional data. For each $t$, we compute the average of the vertices of the directional quantile envelope at the highest $\tau$ for which the directional quantile is not empty and, then, we connect the bivariate medians of each pressure level to obtain the functional median. Visualizing 
variability in bivariate functional data is not trivial, and there is no universal way of visualization of bivariate functional data. We propose a visualization tool for bivariate functional data to visualize the centrality of curves and variability in the curves by characterizing the marginal distributions by directional quantile envelopes and detecting outliers.

To illustrate our visualization tool for bivariate functional quantile envelopes, we divide the radiosonde wind data into two time periods, 1962-1986 and 1987-2011, and estimate the directional quantile envelopes at each pressure level. The estimated directional quantile envelopes for $\tau=0.05,0.0125$, the extreme quantile envelopes for $\tau=0.0002$, the functional median, and the outlying launches are plotted in Figure 2. The directional quantile envelopes give an idea of the shape of the distribution, and we observe that the mid pressure levels have higher variability than the upper and lower pressure levels. From the median curve, it can be observed that the median wind is towards the southeast direction and the wind speed increases as the pressure level drop from 700 to 200 , and then it decreases as the pressure level drops further, with the maximum speed attained at pressure level 200. The launches that had outliers at any of the pressure levels are marked as outlying launches. The first time period, $1962-1986$, contains $0.67 \%$ outliers, while the second time period, $1987-2011$, contains $0.58 \%$ outliers. There are only slight differences in the distributions of two time periods, though they become more apparent in a two-dimensional plot where one pressure level is considered at a time.

\subsubsection{Connected envelopes}

To account for changes in the distribution along $t$, we introduce a method of connecting the directional quantile envelopes along $t$. Each directional quantile envelope is an intersection of the halfspaces corresponding to each direction. However, we do not have a point on the envelope that corresponds to each direction; rather, we have it just for the directions, whose halfspaces lie in the boundary of the envelope. We call such directions active directions. To obtain a point on the envelope corresponding to the directions that are not 
active, i.e., that lie outside the boundary of the envelope, we uniformly distribute the points between two active directions. Now, since we can obtain a point corresponding to each direction on the envelope, we connect the envelopes along each direction, and the connected envelopes characterize the changes along $t$ in all directions.

For radiosonde wind data, connected envelopes are useful to characterize the changes in the distribution of wind along pressure levels, which is often of interest. The connected envelopes for extreme quantiles also provide a way to flag certain potential outlying launches with respect to the observed distribution. Moreover, the proposed method of connected envelopes is different than constructing envelopes at each level and then connecting them. In the proposed methods, the contours at each level borrow information from other pressure levels. If we compute the contours separately at each pressure level, then the contours at each pressure level would be rougher, and it is also not clear on how to connect those separate contours.

Here, we demonstrate connected envelopes using the radiosonde wind data from the second time period, 1987-2011. We obtain a point corresponding to each direction $\boldsymbol{s}$, and connect the points by pressure level. We chose 100 directions to estimate the directional quantile envelopes. We estimate a point corresponding to each of the 100 directions on the envelope and connect the envelopes corresponding to each direction across the eight pressure levels. The connected directional quantile envelopes of the radiosonde wind data for $\tau=0.05$ during the second time period are shown in Figure 3 . At each pressure level, the grey dots represent the observed $u$ and $v$ components of the wind speed, and the black contours are the estimated directional quantile envelopes. The different directions of the connected envelopes are represented by different colors. These connected lines show how the launches behave, for a given direction, at a quantile level of 0.05 or, equivalently, the launches with a Tukey depth of 0.05 . If we increase the quantile level, i.e., increase the depth, then the contours will be smaller and closer to the center, and the connected lines will be closer to the center as well. Similarly, if we estimate the quantile envelopes for a lower quantile level, 
i.e., decrease the depth, the contours will be bigger, and the connected lines for each direction will represent launches with a smaller depth. We can also estimate the connected lines for the tails of the distribution at a very small quantile level by estimating the extreme quantile envelopes.

\subsubsection{Functional outlier detection}

Our proposed method provides quantile envelope estimation for bivariate functional data. Since the quantile based method is robust against outliers, it can be used to identify potential outliers. In Section 2.2.1, we detected the bivariate outliers using extreme quantile envelopes and marked the launches as outlying launches if they had outliers at any pressure level. Here, we present some other approaches of finding functional outliers. Functional outliers can be in the form of magnitude outliers, in which the curves that are distant from the majority of curves are marked as outliers, or shape outliers, where the shape of the curve is different from the shape of the majority of curves in the sample. We suggest two distance measures that might detect some magnitude and shape outliers. If outlier detection is the only purpose rather than visualization, there exist more sophisticated outlier detection procedures in the literature (Hubert et al., 2015; Rousseeuw and Hubert, 2018). To illustrate the two kinds of outliers, we simulate bivariate curves from a mean-zero bivariate Gaussian process with a bivariate Matérn covariance function (as explained in 3.1); then, we contaminate them to simulate a magnitude outlier and a shape outlier, as shown in Figure 4. The curve in red is the shape outlier, while the magnitude outlier is shown in blue.

\section{Magnitude outliers}

To detect magnitude outliers, we need to find the curves that lie at an extreme distance from most of the other curves. We estimate the functional median of the sample using directional quantile envelopes, and find the distance of all the curves from this estimated median curve. Specifically, a sample curve is denoted by $f(t)$, and the median curve is denoted by $f_{\mathrm{M}}(t)$ for ${ }^{t}=t_{1}, \ldots, t_{m}$. The measure of distance used to find the magnitude outliers is given by 
$\mathrm{D}_{\mathrm{M}}(\boldsymbol{f})=\sum_{i=1}^{m} d\left(\boldsymbol{f}\left(t_{i}\right), \boldsymbol{f}_{\mathrm{M}}\left(t_{i}\right)\right)$

where $d$ is the Euclidean distance. We compute the distance measure for all the curves in the sample, and the outliers are marked using a boxplot. To illustrate this method, we simulate 1000 curves from a bivariate Gaussian distribution with mean zero and contaminate $1 \%$ of the curves with magnitude outliers. We estimate the functional median and compute ${ }^{D_{м}}$ for all the curves. We construct the boxplot for $\mathrm{D}_{\mathrm{M}}$; it is shown in Figure 5(a). We observed that all the simulated magnitude outliers could be detected from the boxplot.

\section{Shape outliers}

To detect shape outliers, we need to find the curves with a pattern different from the others. Following similar notations to those used for magnitude outliers, we define a distance measure, which is given by

$$
\mathrm{D}_{\mathrm{S}}(\boldsymbol{f})=\sum_{i=2}^{m} \frac{d\left(\boldsymbol{f}\left(t_{i}\right), \boldsymbol{f}\left(t_{i-1}\right)\right)}{t_{i}-t_{i-1}}
$$

We contaminate $1 \%$ of the curves with shape outliers and compute ${ }^{D_{s}}$ for all the curves. Then, the outliers are marked using a boxplot. Figure 5(b) shows the boxplot for ${ }^{D_{s}}$; all the shape outliers are flagged as such.

\subsection{Quantile Envelope Prediction}

In this section, we propose two methods of predicting the directional quantile envelopes at any new $t^{*}$ within the support of the distribution. To construct a directional quantile envelope, there are two steps: first is to predict the directional quantile in a given direction, second is to take the intersection of the halfspace generated by the predicted directional quantile in all directions. We define two methods to predict the directional quantile in the first step. The first method uses a quantile regression model, while the second method focuses on predicting the directional quantile using univariate kriging.

\subsubsection{Quantile regression model}


We consider the discretized form of the bivariate functional data $\left(X\left(t_{1}\right), \ldots, X\left(t_{m}\right)\right)$, where $\boldsymbol{X}^{X(t)=\left(X_{1}(t), X_{2}(t)\right)}$ for $t \in \in^{\mathcal{T}}$ in $\mathbb{R}^{\mathbb{R}}$. The first method of predicting directional quantile envelopes at a new $t^{*} \in \mathcal{T}$ is based on the quantile regression model (1). For a fixed ${ }^{\tau \in(0,1 / 2]}$, the predicted conditional directional quantile in any direction $s$ can be modeled as $Q_{(\tau, s)}\left(X\left(t^{*}\right)\right)=\beta_{0(\tau, s)}+\hat{b}_{(\tau, s)}\left(t^{*}\right), \quad t^{*} \in \mathcal{T}$.

The predicted conditional directional quantile in direction $\boldsymbol{s}$, denoted by $Q_{(\tau, s)}\left(X\left(t^{*}\right)\right)$, can be obtained by plugging all the estimated parameters into model (1). Once we predict the $\tau^{\text {th }}$ directional quantile in all directions, then we can construct the directional quantile envelope. The predicted directional quantile envelope at $t^{*}$ is given by

$D_{i}(\tau)=\bigcap_{s \epsilon^{\mathcal{S}-1}} H\left(s, Q_{(\tau, s)}\left(\boldsymbol{x}\left(t^{*}\right)\right)\right)$.

Consequently, we can also predict the median at $t^{*}$, by predicting the median envelope at $t^{*}$, for the highest value of $\tau \in(0,1 / 2)$, such that the predicted envelope is not empty.

\subsubsection{Quantile kriging}

In previous studies, kriging models for fixed univariate quantiles have proven effective in building stochastic emulators (Plumlee and Tuo, 2014). Here, we propose a method to obtain bivariate functional quantiles by predicting the $\tau^{\text {th }}$ directional quantile in a direction $s$ at $t^{*}$ via kriging (Matheron, 1963; Cressie, 1993), conditional on the directional quantiles at $t_{1}, \ldots, t_{m}$. If the $\tau^{\tau^{t h}}$ directional quantile in direction $s$ at $t_{i}{ }^{i=1,2, \ldots, m}$, is denoted by ${ }^{Q_{(, s)}\left(X\left(t_{i}\right)\right)}$, then the kriging predictor at $t^{*}$ is given by $Q_{(\tau, s)}\left(\boldsymbol{X}\left(t^{*}\right)\right)=\sum_{i=1}^{m} \lambda_{i} Q_{(\tau, s)}\left(\boldsymbol{X}\left(t_{i}\right)\right)$,

where $\lambda_{i},{ }^{i=1,2, \ldots, m}$ are the kriging weights. The kriging weights are chosen such that the predictor is unbiased, and the mean squared prediction error is 
minimized. For ordinary kriging, the estimated kriging weights $\lambda=\left(\lambda_{1}, \ldots, \lambda_{m}\right)$ are given by

$\lambda=\Sigma^{-1}\left(c+1 \frac{1-1^{T} \Sigma^{-1} \boldsymbol{c}}{\mathbf{1}^{T} \Sigma^{-1} \mathbf{1}}\right)$,

where $^{\Sigma=\operatorname{Cov}\left(\boldsymbol{Q}, \boldsymbol{Q}^{T}\right), \boldsymbol{c}=\operatorname{Cov}\left(\boldsymbol{Q}, Q_{(\tau, s)}\left(\boldsymbol{X}\left(t^{*}\right)\right)\right)}$, and

$\boldsymbol{Q}=\left(Q_{(\tau, s)}\left(\boldsymbol{X}\left(t_{1}\right)\right), \ldots, Q_{(\tau, s)}\left(\boldsymbol{X}\left(t_{m}\right)\right)\right)$. To construct $\Sigma$, we use the Matérn

covariance function. The Matérn correlation is given by

$M(\boldsymbol{h} \mid v, \alpha)=\frac{2^{1-v}}{\Gamma(v)}\left(\frac{(\boldsymbol{h})}{\alpha}\right)^{v} K_{v}\left(\frac{(\boldsymbol{h})}{\alpha}\right)$,

where $K_{v}$ is a modified Bessel function of the second kind, $v$ is the smoothness parameter, and $\alpha$ is the range parameter. The Matérn covariance function is given by $C(\boldsymbol{h})=\sigma^{2} M(\boldsymbol{h} \mid v, \alpha)$, where $\sigma^{2}$ is the marginal variance. The covariance function depends only on the separation vector $h$. The Matérn covariance model can be estimated by ordinary least squares, weighted least squares, or maximum likelihood estimation by assuming a Gaussian random field (Cressie, 1985, 1993; Sherman, 2011). We use univariate kriging to predict the $\tau^{\text {th }}$ directional quantile in direction $s$ at $t^{*}$. Then, the predicted directional quantile envelope for a fixed $\tau \in(0,1 / 2]$ at $t^{*}$ is given by

$$
D_{t^{*}}(\tau)=\bigcap_{s \mathcal{E}^{\mathcal{S}-1}} H\left(s, Q_{(\tau, s)}\left(X\left(t^{*}\right)\right)\right)
$$

We can also predict the median using kriging by predicting the median envelope. Since the quantile kriging method involves the inversion of the covariance matrix, it will be slower than the quantile regression method for larger sample sizes.

\section{Simulation Study}

To illustrate our proposed methods, we conduct two simulation studies in which we predict the directional quantile envelopes using the methods of quantile regression and quantile kriging. We predict the center of the 
distribution using the directional quantile envelope that corresponds to the highest depth, i.e., the median envelope, using different methods and then compare it with the true center of the distribution. In the first simulation, we simulate the curves from a bivariate Gaussian process with mean zero, then contaminate the curves by introducing outliers. In the second simulation, we simulate curves from a model motivated by the radiosonde wind data.

\subsection{Bivariate Gaussian Random Field}

We simulate bivariate functional data $X(t)=\left(X_{1}(t), X_{2}(t)\right)^{T}$ for $t=t_{1}, \ldots t_{m}$ from a bivariate Gaussian random field in ${ }^{\mathbb{R}}$ with mean zero. We assume a stationary full bivariate Matérn covariance model (Gneiting et al., 2010). In a full bivariate Matérn model, the marginal covariance functions are given by

$C_{11}(\boldsymbol{h})=\sigma_{1}^{2} M\left(\boldsymbol{h} \mid v_{1}, \alpha_{1}\right), \quad C_{22}(\boldsymbol{h})=\sigma_{2}^{2} M\left(\boldsymbol{h} \mid v_{2}, \alpha_{2}\right)$,

and the cross-covariance function is given by

$C_{12}(\boldsymbol{h})=C_{21}(\boldsymbol{h})=\rho_{12} \sigma_{1} \sigma_{2} M\left(\boldsymbol{h} \mid v_{12}, \alpha_{12}\right)$,

where ${ }^{M()}$ is of the form (2), $\sigma_{1}^{2}$ and $\sigma_{2}^{2}$ are the marginal variances, $\rho_{12}$ is the cross-correlation, and $v_{12}$ and $\alpha_{12}$ are the cross-covariance smoothness and range parameter, respectively. We simulate the bivariate curves and consider different outlier models. We set $\sigma_{1}=0.1, \sigma_{2}=0.1, \rho_{12}=0.5, v_{1}=1, v_{2}=2$, and $v_{12}=1$, let $\alpha_{1}=\alpha_{2}=\alpha_{12}$, and vary them from 0.1 to 0.5 . The details of the four models used in the simulation are as follows:

1. Model 1: $\boldsymbol{X}(t)=\boldsymbol{e}(t)$, where $\boldsymbol{e}^{\boldsymbol{e}(t)=\left(\mathrm{e}_{1}(t), \mathrm{e}_{2}(t)\right)^{T}}$ is the bivariate Gaussian process in $\mathbb{R}$ with mean zero and full bivariate Matérn covariance model.

2. Model 2: $\boldsymbol{X}(t)=\boldsymbol{e}(t)+\boldsymbol{d}(t), \boldsymbol{d}(t)=\left(s_{1} C, 0\right)$, where $\boldsymbol{C}=4$ indicates the contamination size. Here, $s_{1}=1$ with probability $p$; otherwise $s_{1}=0$. Hence, $s_{1}$ determines the contamination rate. We choose $p=0.01$; therefore, the contamination rate is $1 \%$. 
3. Model 3: Same as Model 2 except $p=0.05$, i.e., $5 \%$ contamination rate..

4. Model 4: Same as Model 2 except $p=0.1$, i.e., $10 \%$ contamination rate.

We generate 1000 curves from each of the four models and for each range parameter, $0.1,0.3$, and 0.5 , at $m=9$ points $(70,100,200,250,300,350$, $400,500,700)$ scaled to $[0,1]$. We predict the center of the distribution at a given $t$, leaving out the data for that particular $t$. We choose ${ }^{t_{6}}=0.4444$ and compare our proposed median estimation to the conditional mean, which is the best predictor for a Gaussian process (Krige, 1981).

We repeat the simulation 100 times and compare the predicted center with the true center of the distribution, which is 0 . We note the mean square predicted error (MSPE) for all four models and for different values of the range parameter. Figure 6 shows the bar plots of MSPE obtained using three methods, the two that we propose here using quantile regression and quantile kriging, and the method of conditional mean, for models with no outliers, $1 \%$ outliers, $5 \%$ outliers, and $10 \%$ outliers, and for three different range parameters, $0.1,0.3$, and 0.5 . In the case of no outliers, MSPE is comparable for the three methods, and all three methods perform well. However, in the case of no outliers, quantile kriging is better than the method of quantile regression. In this simulation setting, quantile kriging is better in prediction since we assume that the directional quantile along the pressure level follows a Gaussian process. When outliers are introduced, the method of the conditional mean is affected the most and performs the worst, while the two methods using quantile regression and quantile kriging are robust to outliers. As the contamination rate increases from $1 \%$ to $10 \%$, this becomes more apparent, as MSPE for the method of conditional mean increases more quickly than for the other two methods. The method of quantile regression is least affected by the outliers, and, in the case of $10 \%$ outliers, the method of quantile regression performs the best. The results are consistent across different range parameters. The computational costs of the two methods were comparable; the cost of the quantile kriging method was slightly higher. The quantile regression method took 32.96 minutes, while quantile kriging took 
37.89 minutes for running 100 simulations. The cost of kriging increases with the number of pressure levels. Therefore, the quantile regression method would be much faster than the kriging method with a high number of pressure levels.

\subsection{Simulation Motivated by Radiosonde Data}

In this simulation design, we simulate curves from the linear models motivated by the radiosonde wind data. The bivariate functional data

$X(t)=\left(X_{1}(t), X_{2}(t)\right)^{T}$, for $t=70,100,200,250,300,400,500,700$, is generated from the linear model

$X_{i}(t)=\beta_{0}+\beta_{1} D_{i}+b(t)+\epsilon_{i}, \quad i=1,2,(3)$

where $b(t)$ is the cubic spline function given by $b(t)=\beta_{1} t+\beta_{2} t^{2}+\beta_{3} t^{3}+\sum_{j=1}^{K} \delta_{j}\left(t-k_{j}\right)_{+}^{3}$ and $\left\{k_{j}\right\}_{j=1}^{K}$ are a prespecified set of knots.

$D_{i}$ is a dummy variable: $D_{i}=0$ if $i=1$ (u-component of wind) and $D_{i}=1$ if $i=2$ ( $v$-component of wind). First, we fit this spline model (3) to the radiosonde wind data and obtain the estimated coefficients. Then, we generate data using the estimated coefficients, with ${ }^{\epsilon}{ }_{1}$ and ${ }^{\epsilon}{ }_{2}$ assumed to be independently simulated from a skew normal distribution with the shape parameter (denoted by $\alpha$ ) being, $0,1,2$, and 3 .

We simulate 1000 curves at eight pressure levels: 70, 100, 200, 250, 300, 400,500 , and 700 . At pressure level 300 , we leave out the data and predict the median using the three methods, quantile kriging, quantile regression, and the conditional mean. Then, we compare them with the truth. We repeat this simulation 100 times and compute MSPE. The MSPE results from the four models that correspond to the four shape parameters $(\alpha=0,1,2$, and 3$)$ are listed in Table 1 for the three methods. The quantile kriging method performs the best, followed by the method of quantile regression, and then conditional mean. In the case of normally distributed errors (i.e., in the case of $\alpha=0$ ), all three methods perform reasonably well and are comparable. However, as the skewness of the distribution increases (i,e., as $\alpha$ increases), the method of 
conditional mean performs poorly, while the quantile kriging and quantile regression methods are still robust. We have done other simulations with more functional data points, and the results are similar. The simulation designs we show is to mimic the available radiosonde data.

\section{Applications to Radiosonde Winds}

To demonstrate our methods, we use radiosonde wind data from a station in Denver, Colorado. The radiosonde wind data consists of two wind components, i.e., $u$-component and $v$-component, obtained by transforming polar coordinates of wind speed and direction to cartesian coordinates. We consider a set of 35,816 launches, which were recorded twice a day from March 3, 1962, to October 31, 2011, along eight standard atmospheric pressure levels, $70,100,200,250,300,400,500$, and $700 \mathrm{hPa}$. The estimated directional quantile envelopes for the bivariate radiosonde wind data from two periods have been discussed in Section 2.2.2. In this section, we apply the methods of predicting quantile envelopes that were discussed in Section 2.3 with the methods of quantile regression and quantile kriging. We also estimate the connected envelopes to characterize the changes across pressure levels.

\subsection{Prediction of Median}

In this section, we predict the center of the bivariate distribution of radiosonde winds at each pressure level using the methods of quantile kriging, quantile regression, and conditional mean. First, we center the observations from each pressure level to eliminate the potential trend along the pressure level, by removing their respective bivariate Tukey median, which is a robust estimator of the center. Then, we perform kriging along the pressure level, assuming zero mean. We predict the median using quantile kriging and quantile regression, and compare it with the estimated median of the observed data using the leave-one-out cross-validation approach. We also compute the conditional mean and compare it to the observed median. We leave one pressure level out at a time and compute the MSPE in the following way: 
$\operatorname{MSPE}=\frac{1}{p} \sum_{i=1}^{p} d\left(\boldsymbol{m}_{i}, \hat{\boldsymbol{m}}_{i}\right)$,

where $d$ is the Euclidean distance, ${ }^{\boldsymbol{m}_{i}}$ and $\hat{\boldsymbol{m}}_{i}$ are the true and predicted medians of the $i^{\text {th }}$ pressure level, respectively, and $p$ is the number of pressure levels left out. We leave out each of the pressure levels 100, 200, 250,300 , and 400 , one at a time. The MSPE results for both time periods, and the three methods are shown in Table 2. For both the time periods, we observe that the method of quantile kriging performs best. For the first time period, the methods of quantile regression and conditional mean are comparable, with the conditional mean method performing slightly better, while for the second time period, the quantile regression method performs better than the conditional mean method. Therefore, the proposed method can predict the center of the distribution of radiosonde winds robustly for skewed and heavy-tailed data and provide summary statistics for the bivariate distribution of components of the wind.

\subsection{Predicting Envelope for a Given $\boldsymbol{T}$}

Above, we demonstrated the application of quantile envelope prediction by predicting the median using methods based on quantile kriging, quantile regression, and the conditional mean. Here, we predict the envelopes for any $\tau \in[0,1 / 2)$. We predict the distribution of the bivariate distribution of wind at unknown pressure levels, using the information recorded at the standard atmospheric pressure levels from the radiosonde wind data. We illustrate the prediction of quantile envelopes for a given pressure level and for a fixed $\tau$ by predicting the quantile envelope at each of the known pressure levels and then comparing it with the envelope estimated from the observations at the corresponding pressure level. We take the pressure levels 200 and 300 for $\tau=0.05$ as an example. Figure 7 shows scatter plots between the $u$ and $v$ components of the radiosonde wind at the pressure levels 200 and 300 . The quantile envelopes predicted by quantile kriging and quantile regression are shown in green and red, respectively, and the observed envelope in blue. The predictions of both methods are reasonably good, as they are very close to 
the observed envelope for both the pressure levels. These methods can be used to predict the bivariate distribution of wind in parts of the atmosphere where no radiosonde observations are available.

It is difficult to determine visually from Figure 7 which method performs better. In Section 2.2.3, we defined the idea of connected envelopes, where we obtain a point on an envelope corresponding to each direction. Using the connected envelopes, we compare the predicted quantile envelopes from both the methods numerically. We obtain MSPE as

M S PE $=\frac{1}{100} \sum_{i=1}^{100} d\left(\boldsymbol{p}_{s_{i}}, \hat{\boldsymbol{p}}_{s_{i}}\right)$

where $\boldsymbol{p}_{s_{i}}$ and $\hat{\boldsymbol{p}}_{s_{i}}$ are the points on an envelope corresponding to the direction $s_{i}, i=1,2, \ldots, 100$, and $d$ is the Euclidean distance. We estimate the quantile envelopes for the pressure levels $100,200,250,300$, and 400 for $\tau=0.05$, and compare them to the observed envelope to obtain MSPE. Table 3 shows MSPE obtained using both methods. Overall, the quantile kriging method has a better performance. However, the quantile regression method performs better in some cases, such as the mid pressure levels. For instance, the quantile kriging method performs better for pressure level 200, while the quantile regression method is better for pressure level 300 .

\section{Discussion}

In this paper, we define a model for estimating and predicting the directional quantile envelopes of bivariate functional data, $X(t)=\left(X_{1}(t), X_{2}(t)\right)^{T}$, where $t$ belongs to an interval ${ }^{\mathcal{T}}$ in ${ }^{\mathbb{R}}$, and extend the existing approaches for multivariate data. The functional aspect allows us to connect or predict those quantiles continuously along $t$. These directional quantile envelopes completely characterize the distribution marginally for each $t$. The proposed nonparametric approach is suitable for estimating bivariate distributions that are non-Gaussian and skewed. We use the proposed model to estimate the directional quantile envelopes for the $u$ and $v$ components of radiosonde wind data across several atmospheric pressure levels. 
The developed visualization tool reflects the geometry of bivariate functional data with the help of the estimated directional quantile envelopes, functional median, and outlier detection. We visualize the geometry of the radiosonde wind data at eight standard atmospheric pressure levels by estimating the directional quantile envelopes at three quantile levels and estimating its functional median. To flag outliers and errors in the radiosonde measurements, we estimate the extreme quantile envelopes and detect the outliers at each pressure level. We define two methods of detecting two types of functional outliers, both magnitude and shape outliers.

We also define two methods of predicting directional quantile envelopes at a given $t$ for bivariate functional data. It provides a way of obtaining the bivariate conditional distributions of non-Gaussian processes. The first method uses quantile regression to model the $\tau^{\text {th }}$ directional quantile, and the second uses a kriging approach to predict the $\tau^{\text {th }}$ directional quantile at a new $t$. Using simulation studies, we show that both methods are robust against outliers and skewed distributions. Overall, the method of quantile kriging performed better, but there were some cases (e.g., Gaussian random field with $10 \%$ outliers) when the quantile regression method worked better. Both of these proposed methods provide a robust prediction over the conditional mean.

The $\mathrm{R}$ code for the methods described in this paper has been made publicly available on https://github.com/agarwag/Bivariate-Functional-QuantileEnvelopes. The readers can use the methods to visualize bivariate functional data and estimate even non-Gaussian, skewed, heavy-tailed distributions.

\section{Acknowledgement}

The research reported in this publication was supported by funding from King Abdullah University of Science and Technology (KAUST), under award number OSR-2019-CRG7-3800. The dataset used in this research was provided by the Research Data Archive at the National Center for Atmospheric Research (NCAR), Computational and Information Systems Laboratory. 


\section{References}

Barnett, V. (1976). The ordering of multivariate data. Journal of the Royal Statistical Society. Series A (General), pages 318-355.

Chakraborty, B. (2001). On affine equivariant multivariate quantiles. Annals of the Institute of Statistical Mathematics, 53(2):380-403.

Chakraborty, B. (2003). On multivariate quantile regression. Journal of Statistical Planning and Inference, 110(1):109-132.

Chaudhuri, P. (1996). On a geometric notion of quantiles for multivariate data Journal of the American Statistical Association, 91(434):862-872

Chien, H. and Smith, P. J. (1973). On the estimation of kinematic parameters in the atmosphere from radiosonde wind data. Mon. Wea. ReV, 101:252-261.

Claeskens, G., Hubert, M., Slaets, L., and Vakili, K. (2014). Multivariate functional halfspace depth. Journal of the American Statistical Association, 109(505):411-423

Cressie, N. (1985). Fitting variogram models by weighted least squares. Journal of the International Association for Mathematical Geology, 17(5):563586

Cressie, N. A. (1993). Statistics for spatial data. Wiley series in probability and mathematical statistics. Applied probability and statistics section.

Dai, W. and Genton, M. G. (2018a). Functional boxplots for multivariate curves. Stat, 7:e190.

Dai, W. and Genton, M. G. (2018b). Multivariate functional data visualization and outlier detection. Journal of Computational and Graphical Statistics, 27(4):923-934

Dai, W. and Genton, M. G. (2019). Directional outlyingness for multivariate functional data. Computational Statistics \& Data Analysis, 131:50-65. 
(2014). Ncar upper air database, 1920-ongoing. Retrieved from: http://rda.ucar.edu/datasets/ds370.1/.

Durre, I., Vose, R. S., and Wuertz, D. B. (2006). Overview of the integrated global radiosonde archive. Journal of Climate, 19(1):53-68.

Fraiman, R. and Muniz, G. (2001). Trimmed means for functional data. Test, 10(2):419-440

Gneiting, T., Kleiber, W., and Schlather, M. (2010). Matérn cross-covariance functions for multivariate random fields. Journal of the American Statistical Association, 105(491):1167-1177

Hallin, M., Paindaveine, D., and Siman, M. (2010). Multivariate quantiles and multiple-output regression quantiles: From $l_{1}$ optimization to halfspace depth. Ann. Statist., 38(2):635-669.

Hollingsworth, A. and Lönnberg, P. (1986). The statistical structure of shortrange forecast errors as determined from radiosonde data. part $\mathrm{i}$ : The wind field. Tellus A, 38(2):111-136

Hubert, M., Rousseeuw, P. J., and Segaert, P. (2015). Multivariate functional outlier detection. Statistical Methods \& Applications, 24(2):177-202.

Kalnay, E., Kanamitsu, M., Kistler, R., Collins, W., Deaven, D., Gandin, L., Iredell, M., Saha, S., White, G., and Woollen, J. (1996). The ncep/ncar 40year reanalysis project. Bulletin of the American meteorological Society, 77(3):437-472

Kanamitsu, M., Ebisuzaki, W., Woollen, J., Yang, S.-K., Hnilo, J., Fiorino, M., and Potter, G. (2002). Ncep-doe amip-ii reanalysis (r-2). Bulletin of the American Meteorological Society, 83(11):1631-1644

Koenker, R. and Bassett Jr, G. (1982). Robust tests for heteroscedasticity based on regression quantiles. Econometrica: Journal of the Econometric Society, pages $43-61$ 
Kong, L. and Mizera, I. (2012). Quantile tomography: using quantiles with multivariate data. Statistica Sinica, pages 1589-1610.

Kong, L., Shu, H., Heo, G., and He, Q. C. (2015). Estimation for bivariate quantile varying coefficient model. arXiv preprint arXiv:1511.02552.

Kong, L. and Zuo, Y. (2010). Smooth depth contours characterize the underlying distribution. Journal of Multivariate Analysis, 101(9):2222-2226.

Krige, D. G. (1981). Lognormal-de Wijsian geostatistics for ore evaluation. South African Institute of mining and metallurgy Johannesburg.

Liu, R. Y. (1990). On a notion of data depth based on random simplices. The Annals of Statistics, pages 405-414.

López-Pintado, S. and Romo, J. (2009). On the concept of depth for functional data. Journal of the American Statistical Association, 104(486):718-734

López-Pintado, S. and Romo, J. (2011). A half-region depth for functional data. Computational Statistics \& Data Analysis, 55(4):1679-1695.

López-Pintado, S., Sun, Y., Lin, J. K., and Genton, M. G. (2014). Simplicial band depth for multivariate functional data. Advances in Data Analysis and Classification, 8(3):321-338 1862-5347.

Matheron, G. (1963). Principles of geostatistics. Economic geology, 58(8):1246-1266.

Moors, J., Wagemakers, R. T. A., Coenen, V., Heuts, R., and Janssens, M. (1996). Characterizing systems of distributions by quantile measures. Statistica Neerlandica, 50(3):417-430

Oja, H. (1983). Descriptive statistics for multivariate distributions. Statistics \& Probability Letters, 1(6):327-332.

Plumlee, M. and Tuo, R. (2014). Building accurate emulators for stochastic simulations via quantile kriging. Technometrics, 56(4):466-473 
Rousseeuw, P. J. and Hubert, M. (2018). Anomaly detection by robust statistics. Wiley Interdisciplinary Reviews: Data Mining and Knowledge Discovery, 8(2):e1236

Serfling, R. (2002). A depth function and a scale curve based on spatial quantiles, pages 25-38. Springer.

Sherman, M. (2011). Spatial statistics and spatio-temporal data: covariance functions and directional properties. John Wiley \& Sons.

Sherwood, S. C., Meyer, C. L., Allen, R. J., and Titchner, H. A. (2008). Robust tropospheric warming revealed by iteratively homogenized radiosonde data. Journal of Climate, 21(20):5336-5352.

Sherwood, S. C. and Nishant, N. (2015). Atmospheric changes through 2012 as shown by iteratively homogenized radiosonde temperature and wind data (iukv2). Environmental Research Letters, 10(5):054007

Struyf, A. J. and Rousseeuw, P. J. (1999). Halfspace depth and regression depth characterize the empirical distribution. Journal of Multivariate Analysis, 69(1):135-153.

Sun, Y. and Genton, M. G. (2011). Functional boxplots. Journal of Computational and Graphical Statistics, 20(2):316-334

Sun, Y. and Genton, M. G. (2012). Adjusted functional boxplots for spatiotemporal data visualization and outlier detection. Environmetrics, 23(1):54-64

Sun, Y., Hering, A. S., and Browning, J. M. (2017). Robust bivariate error detection in skewed data with application to historical radiosonde winds. Environmetrics, 28(3):e2431

Tukey, J. W. (1975). Mathematics and the picturing of data. In Proceedings of the International Congress of Mathematicians, volume 2, pages 523-531. 
Vardi, Y. and Zhang, C.-H. (2000). The multivariate I1-median and associated data depth. Proceedings of the National Academy of Sciences, 97(4):14231426

Wei, Y. (2008). An approach to multivariate covariate-dependent quantile contours with application to bivariate conditional growth charts. Journal of the American Statistical Association, 103(481):397-409

Zuo, Y. (2003). Projection-based depth functions and associated medians. Ann. Statist., 31(5):1460-1490.

Zuo, Y. and Serfling, R. (2000). General notions of statistical depth function, Ann. Statist., 28(2):461-482. 
Pressure level: 70

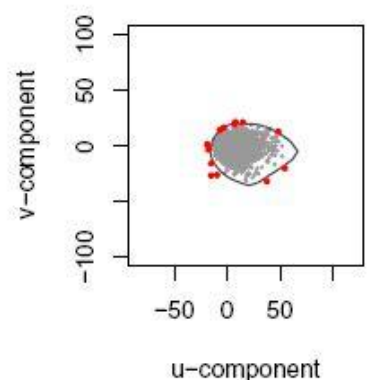

Pressure level: 300

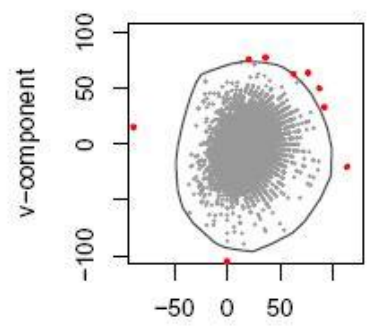

u-component
Pressure level: 100

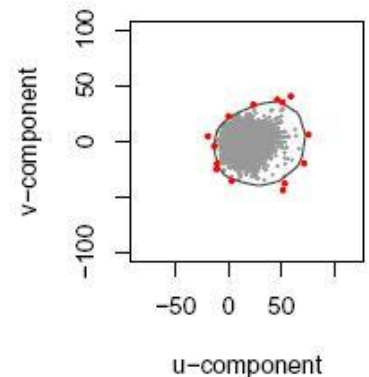

Pressure level: 400

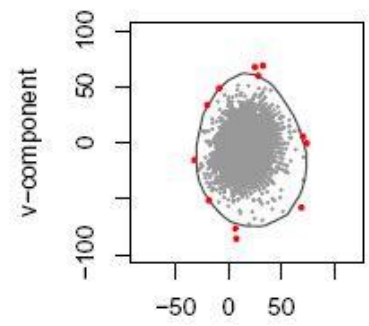

u-component
Pressure level: 200

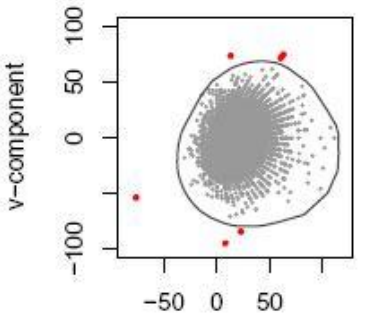

u-component

Pressure level: 500

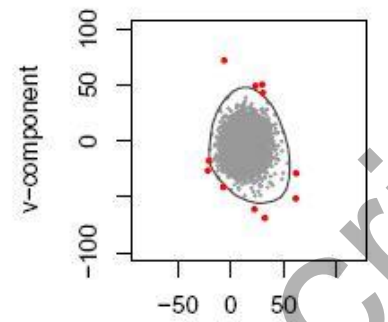

u-component
Pressure level: 250

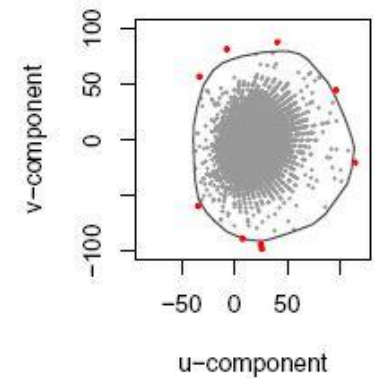

Pressure level: 700

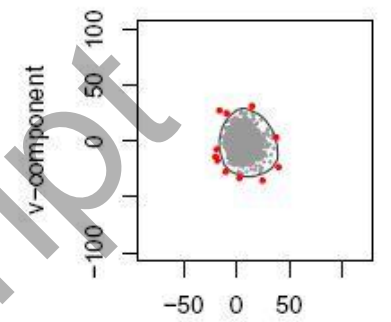

u-component

Fig. 1 Each of the eight panels shows a scatter plot of the $u$ and $v$ components of the radiosonde wind data and the estimated extreme quantile envelopes for $\tau=0.0002$ at a given pressure level. The outliers are marked by red dots. 

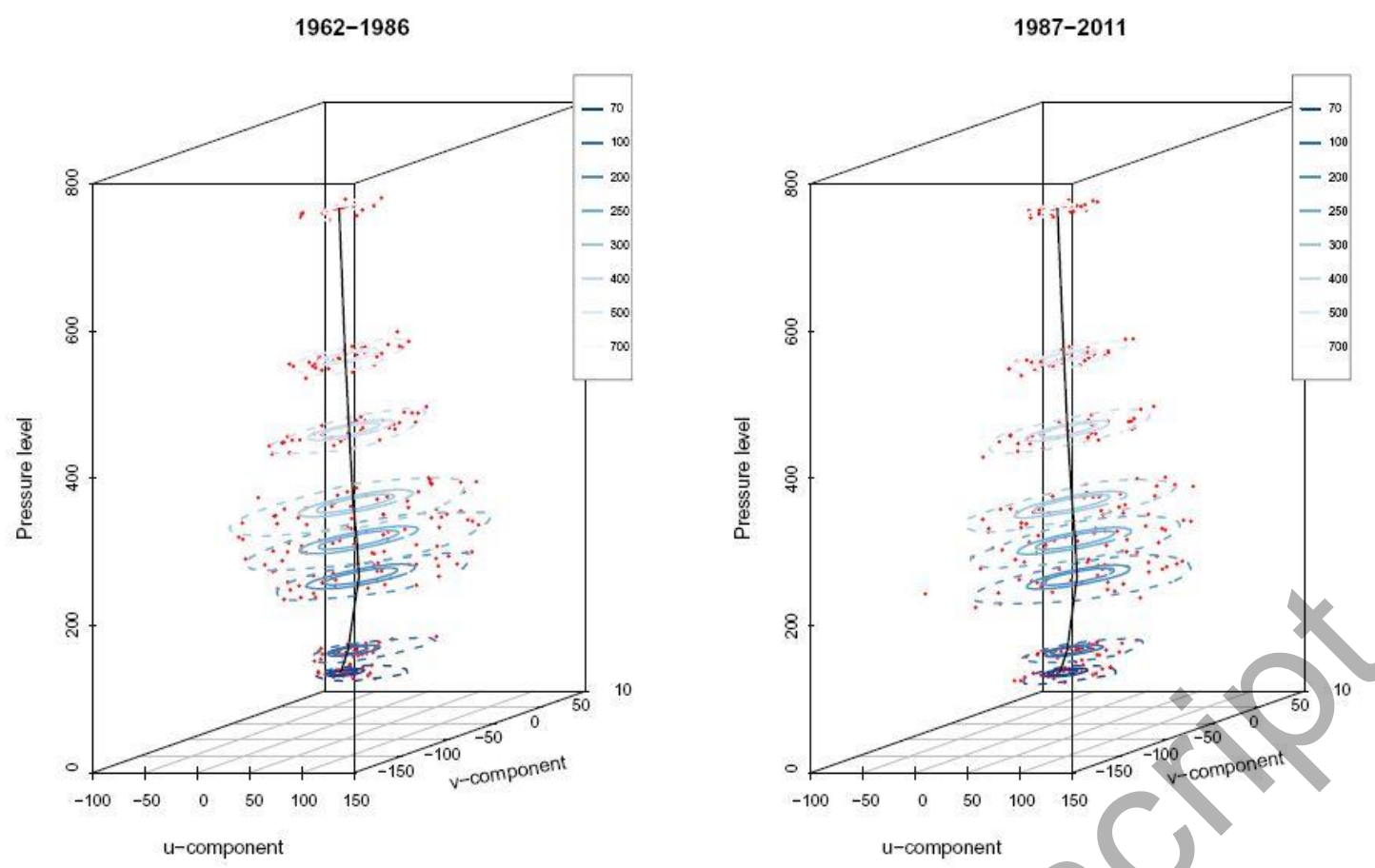

(a)

Fig. 2 The solid contours are the estimated directional quantile envelopes for $\tau=0.05,0.0125$, and the dashed contours are the extreme quantile envelopes; the color variation represents the different pressure levels. The red dots are the outlying launches. The solid black line is the estimated functional median. (a) First time period 1962-1986, and (b) second time period 1987-2011. 


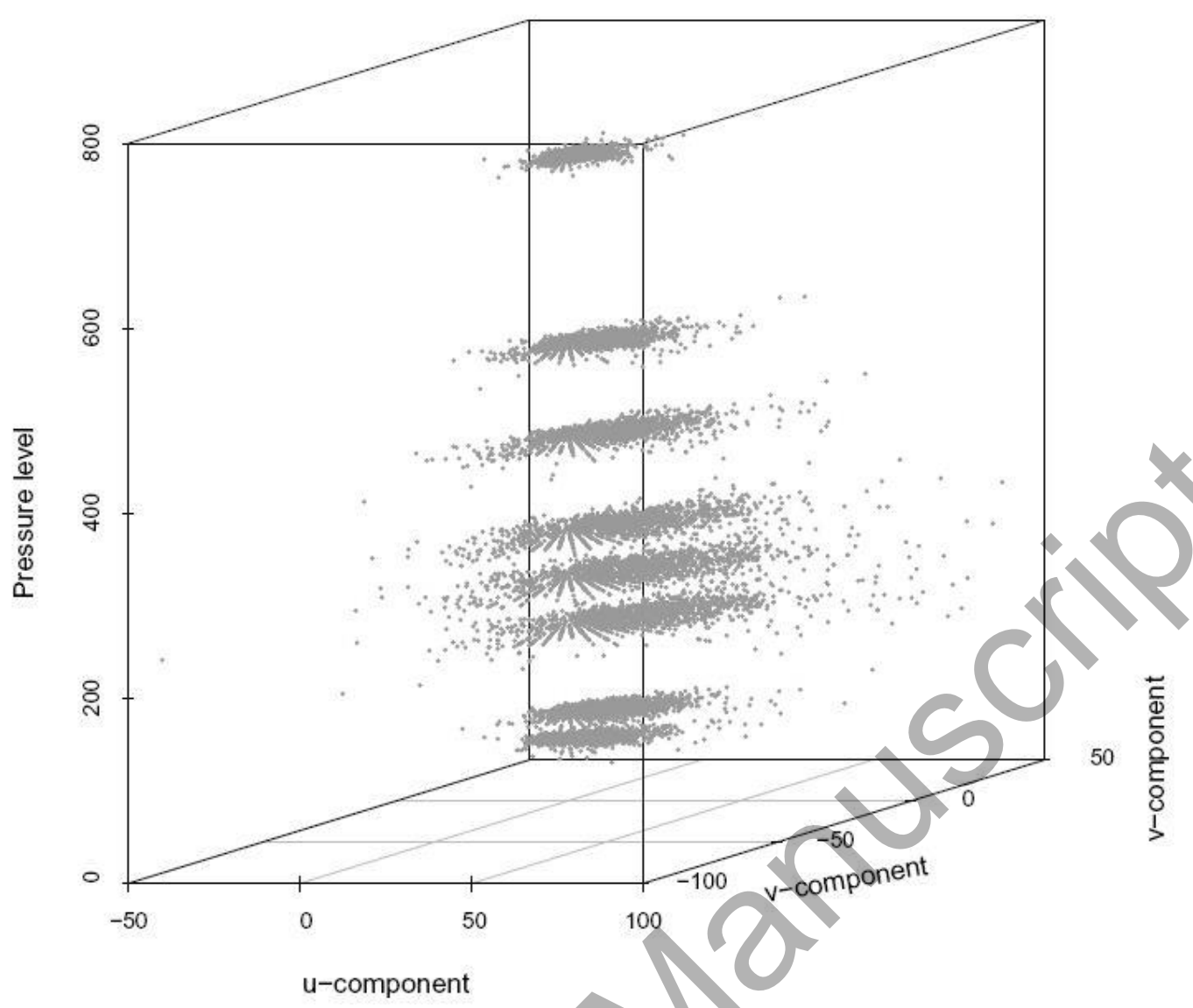

Fig. 3 The three-dimensional plot represents the $u$ component on the x-axis, the $v$ component on the $y$-axis, and the pressure level on the z-axis. The grey dots represent the observed $u$ and $v$ components of wind speeds from the radiosonde data, and the black contours represent the estimated directional quantile envelopes for $\tau=0.05$ during the time period 1987-2011. The different colors of the connected directional quantile envelopes for $\tau=0.05$ represent different directions. 


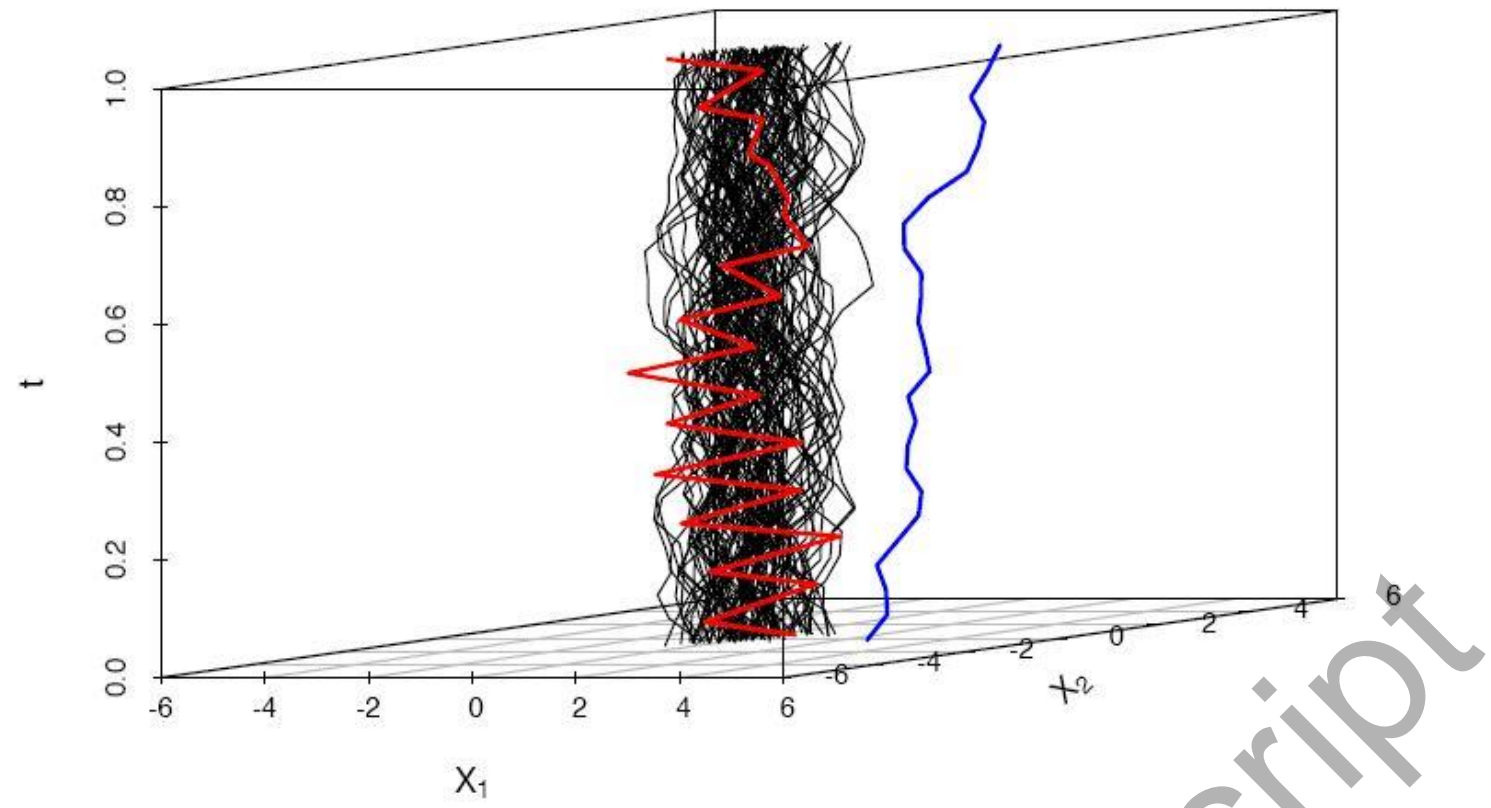

Fig. 4 Simulated curves from a bivariate Gaussian distribution with zero mean. The curve in red is the shape outlier; the curve in blue is the magnitude outlier. 


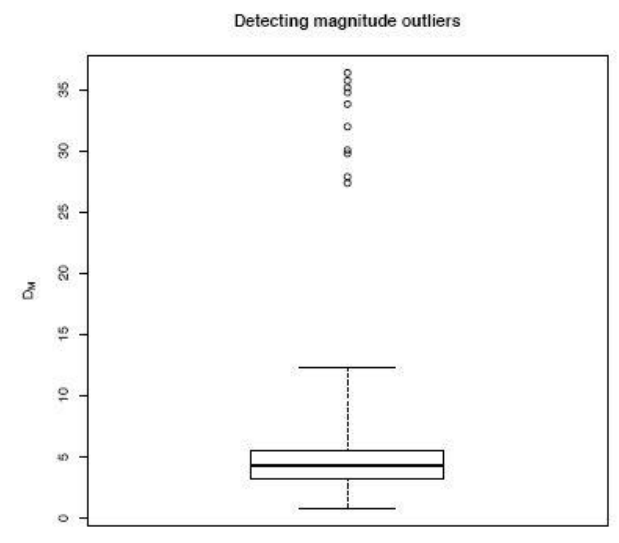

(a)

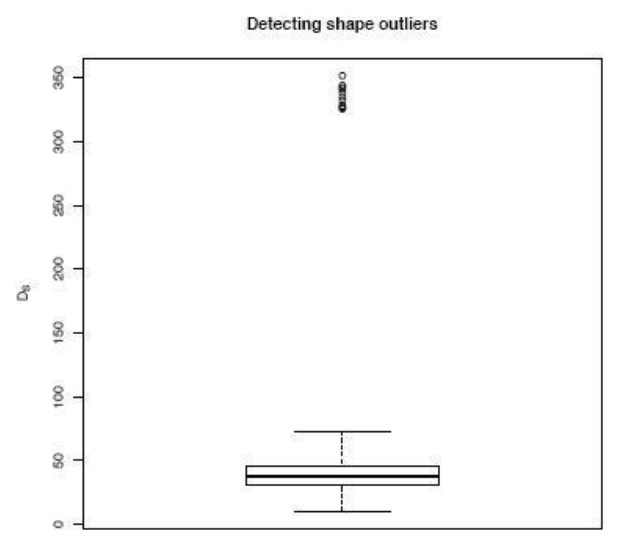

(b)

Fig. 5 Boxplots to detect functional outliers. (a) Magnitude outliers and (b) shape outliers. 

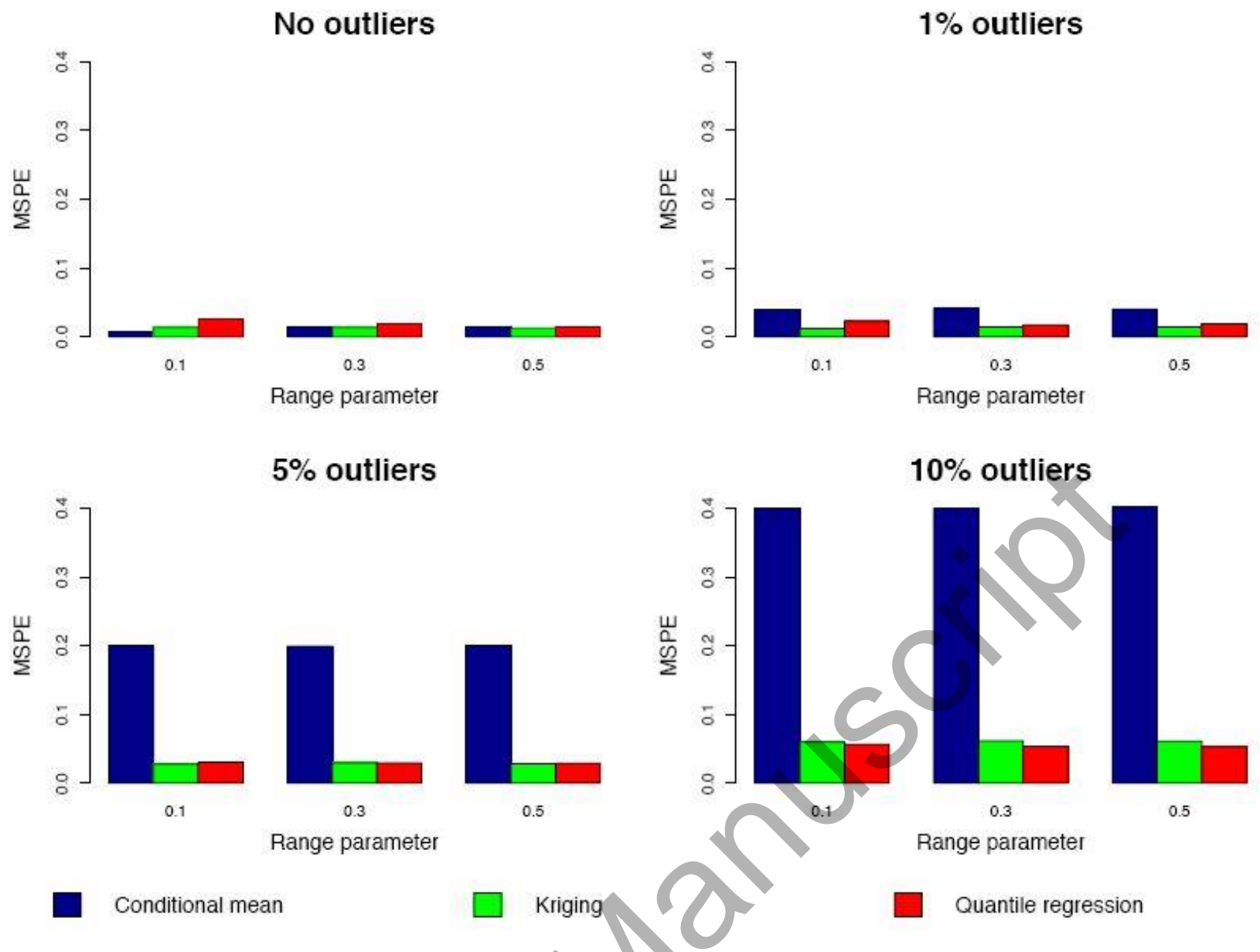

Fig. 6 Results of simulations from a bivariate Gaussian process. The four panels represent the models with no outliers, $1 \%$ outliers, $5 \%$ outliers, and $10 \%$ outliers. Each panel shows bar plots of MSPE for the three methods, conditional mean (blue), quantile kriging (green), quantile regression (red), and for three cases of range parameters, $0.1,0.3$, and 0.5 . 


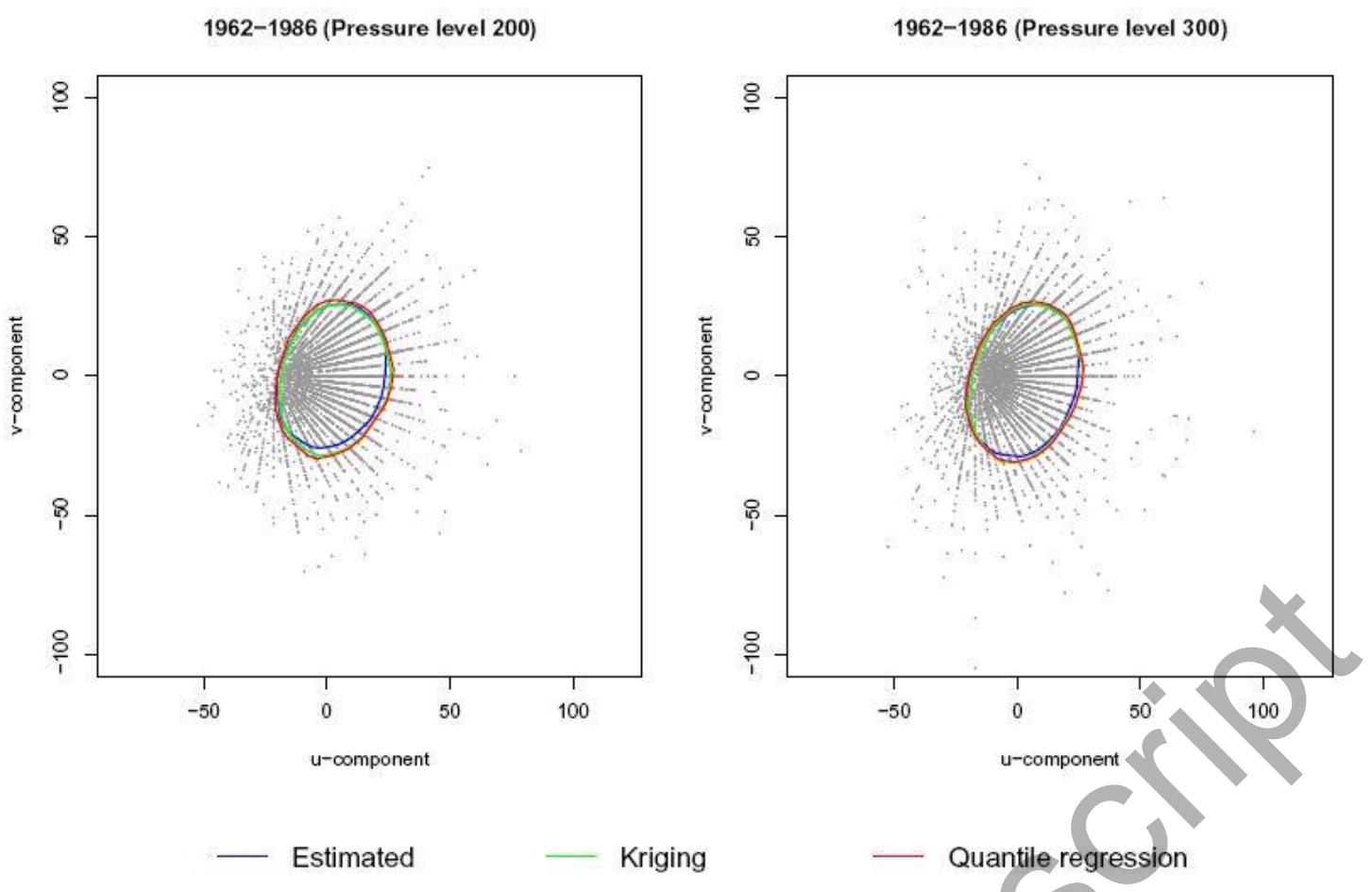

Fig. 7 The plots show the scatter between the $u$-component and $v$-component of the wind along the with the estimated quantile envelope (blue) from the observed data and the predicted quantile envelopes from the method of kriging (green) and quantile regression (red). The left panel shows the plot for pressure level 200 and the right panel represents pressure level 300. 
Table 1 Mean square error from model motivated by real data.

\begin{tabular}{||c|c|c|c|c|}
\hline Method $\backslash \alpha$ & 0 & 1 & 2 & 3 \\
\hline \hline Quantile kriging & 0.2453 & 0.2448 & 0.2455 & 0.2482 \\
\hline Quantile regression & 0.3083 & 0.3157 & 0.2902 & 0.3144 \\
\hline Conditional mean & 0.2896 & 1.1008 & 1.2979 & 1.3744 \\
\hline \hline & & & & \\
\hline
\end{tabular}

Table 2 MSPE obtained by comparing the center of the radiosonde wind data distribution using leave-one-out cross-validation over two time periods.

\begin{tabular}{|c|c|c|}
\hline Method & $\mathrm{MSPE}_{1}$ & MSPE $_{2}$ \\
\hline Quantile kriging & 0.2343 & 0.2224 \\
\hline Quantile regression & 0.9318 & $\mid 0.7877$ \\
\hline Conditional mean & 0.9267 & 0.9431 \\
\hline
\end{tabular}


Table 3 MSPE obtained by comparing envelopes at each direction of the two methods kriging and quantile regression from the observed envelope.

\begin{tabular}{|l|l|l||}
\hline Pressure level & Quantile kriging & Quantile regression \\
\hline 100 & 2.6881 & 7.4713 \\
\hline \hline 200 & 2.8423 & 4.0829 \\
\hline 250 & 2.5331 & 2.3622 \\
\hline 300 & 3.4074 & 3.1900 \\
\hline 400 & 4.0831 & 6.4693 \\
\hline \hline & & \\
\hline
\end{tabular}

